\title{
Anti-inflammatory polymer electrodes for glial scar treatment: bringing the conceptual idea to future results
}

\author{
Maria Asplund ${ }^{1,2,3 *}$, Christian Boehler ${ }^{1,2,3}$ and Thomas Stieglitz ${ }^{1,3}$ \\ Biomedical Microtechnology, IMTEK, Albert-Ludwigs Universität, Freiburg, Germany \\ ${ }^{2}$ Freiburg Institute for Advanced Studies FRIAS, Albert-Ludwigs Universität, Freiburg, Germany \\ ${ }^{3}$ BrainLinks-BrainTools Cluster of Excellence, Albert-Ludwigs Universität, Freiburg, Germany
}

\section{Edited by:}

Ulrich G. Hofmann

Albert-Ludwigs-University Freiburg,

Germany

\section{Reviewed by:}

Pascal Darbon, Université de

Strasbourg, France

Fabrice O. Morin, Fatronik-Tecnalia

Foundation, Spain

${ }^{*}$ Correspondence:

Maria Asplund, Biomedical

Microtechnology, IMTEK,

Albert-Ludwigs Universität,

Georges-Köhler Allee 102, Freiburg

79108, Germany

e-mail:maria.asplund@imtek.de
Conducting polymer films offer a convenient route for the functionalization of implantable microelectrodes without compromising their performance as excellent recording units. A micron thick coating, deposited on the surface of a regular metallic electrode, can elute anti-inflammatory drugs for the treatment of glial scarring as well as growth factors for the support of surrounding neurons. Electro-activation of the polymer drives the release of the substance and should ideally provide a reliable method for controlling quantity and timing of release. Driving signals in the form of a constant potential (CP), a slow redox sweep or a fast pulse are all represented in literature. Few studies present such release in vivo from actual recording and stimulating microelectronic devices. It is essential to bridge the gap between studies based on release in vitro, and the intended application, which would mean release into living and highly delicate tissue. In the biological setting, signals are limited both by available electronics and by the biological safety. Driving signals must not be harmful to tissue and also not activate the tissue in an uncontrolled manner. This review aims at shedding more light on how to select appropriate driving parameters for the polymer electrodes for the in vivo setting. It brings together information regarding activation thresholds for neurons, as well as injury thresholds, and puts this into context with what is known about efficient driving of release from conducting polymer films.

Keywords: conducting polymer, dexamethasone, drug delivery, glial scarring, neural interfaces

\section{INTRODUCTION}

Recent advances in the field of conducting polymers point out their potential as drug delivery coatings from the surfaces of microelectrodes. This is of interest considering it comprises an opportunity to target cells in the close vicinity of an implant with high spatial and temporal control of release. Glial scarring is a physiological process that deteriorates electrode function by forming a substantial barrier for signal transduction. Persistent inflammation, following the scarring process, is believed to be the reason why neurons are lost at the site of the implant further complicating high resolution in recording and stimulation (Turner et al., 1999; Szarowski et al., 2003; Biran et al., 2005). Systemic treatment using anti-inflammatory drugs such as Dexamethasone (Dex) has been suggested as a possible strategy for facilitating close integration of the implant with neural tissue (Spataro et al., 2005). Conducting polymer electrodes designed to elute drugs upon electro-activation are an alternative to systemic treatment of glial scarring (Abidian et al., 2006; Wadhwa et al., 2006; Evans et al., 2009; Richardson et al., 2009; Luo and Cui, 2009a,b; Stevenson et al., 2010; Yue et al., 2013). Polymers in question are mainly Polypyrrole (PPy), poly(3,4-ethylene dioxythiophene) (PEDOT) but more recently also polyterthiophene (PTTh) has been suggested as a candidate (Stevenson et al., 2010). This functionality is until now almost exclusively studied in vitro. Here we discuss this intriguing possibility, its requirements in terms of electronic control of the implant, and the restrictions set by electrochemical safety limits, to form the basis for continued investigations in vivo.

Electro-activation is essential for triggering release but cannot be allowed to cause detrimental effects on the cellular microenvironment. The conducting polymer electrode is not analogous to a metallic electrode and direct currents can, and must, to some extent be tolerated to drive drugs out of the electrode. On the other hand, one must not overlook the possibility that byproducts form in body fluids as a result of electro-activation, or that the neuronal circuitry is unintentionally activated, which means that potentials must be kept within strict boundaries. The solution that comes close at hand is to ensure that signals are maintained below the activation threshold. Then the question boils down to if this type of signal can be used to drive ionic drugs out of the electrode? The majority of studies focus on release by cyclic voltammetry (CV), a signal sufficiently slow to give room for diffusion limited processes to contribute, in contrast to fast stimulation pulses, generally designed to employ primarily capacitive effects.

Studies show that release can be precisely managed by the appropriate electrochemical driving signal. However, the means to keep exact control of charge and voltage are limited in an implant where three-electrode electrochemical systems are rarely implemented. Furthermore, the circuitry designed for stimulation in vivo cannot necessarily accommodate the same type of measurements and control as the electrochemical potentiostat. 
Therefore, electronics and implantable reference electrodes that meet this requirement need to be developed. If glial scarring is really to be treated by the suggested method it cannot come at the cost of connecting lab animals to fully functional external potentiostats but the solution must come as a miniaturized implant.

Finally we intend to outline the possibilities in terms of quantities of drugs that can be delivered and, to some extent, the variety of drugs that could come in question. Most studies focus on delivery of the anti-inflammatory drug Dex but results point out that other drugs with similar size and charge could also be potential candidates.

In summary, we present the possibilities of conducting polymer based release for glial scar treatment. Benefits of the method will be put in perspective with design challenges that have to be met from the electronics side. This information is essential for enabling more studies to proceed to the implant stage, shedding light on how to make the best out of this novel and exciting concept.

\section{ELECTRODEPOSITION OF CONDUCTING POLYMERS}

Conducting polymers can be deposited on top of microelectrodes using an aqueous electrodeposition process. The reaction is driven in a supporting electrolyte in which the monomer (M) is dissolved or dispersed together with appropriate counter ions (CI). The monomers oxidized at the surface of the working electrode build up an insoluble layer of conducting polymer on its surface. To maintain charge neutrality, the negatively charged CI are at the same time electrostatically entrapped in the formed material according to the following reaction:

$$
M+C I^{-} \stackrel{\text { oxidize }}{\longrightarrow}(M)^{+} C I^{-}
$$

The nature of the counter ion is decisive for the ionic exchange properties of the formed polymer (Bobacka et al., 2000; Jager et al., 2000). The CI can be small or large, inorganic or organic molecules, or a combination of several different negatively charged molecules can be included in the supporting electrolyte to form a more complex material. If a biologically relevant molecule is used as counter ion, the formed polymer can be biofunctionalized since the molecule is efficiently entrapped in the porous polymer matrix yet still available for reactions on the surface of the polymer material (Asplund et al., 2008). In addition it can be released from the polymer upon altering the polymer redox state.

There are some restrictions in the choice of biological ions that can come in question for the counter ion incorporation technique. The one step approach described above would work exclusively for negatively charged biomolecules. For the delivery functionality to be efficient, a further constraint is that the molecule must be sufficiently small to be able to diffuse through the polymer matrix. It is difficult to give a precise estimate on what could be considered as sufficiently small, since the porosity is not an absolute property but can be influenced by the electrodeposition process. To give some figure of merit, successful release has been shown for molecules up to the range of $0.5 \mathrm{kDa}$. Although some authors report release of substantially larger substances such as protein fragments, it is clear that this is more challenging and might primarily rely on actuation of the polymer material rather than electrostatic binding and release (Thompson et al., 2006; Evans et al., 2009; Richardson et al., 2009). This topic is discussed in detail in section Release Systems and Mechanisms.

As an alternative to the direct incorporation in Equation 1, an ion exchange approach can be used to drive ions into the already formed polymer film for subsequent release upon reversing the potential (Xiao et al., 2007). Furthermore, a carrier phase can be introduced into the material by allowing the polymer to form within a network of beads or fibers already containing the substance to be delivered (Abidian et al., 2006; Luo et al., 2011). A non-charged substance in the larger size range, which is made available in the supporting electrolyte, can be adsorbed to the surface in parallel with the deposition process and thereby also be mechanically entrapped although not electrostatically bound (Asplund et al., 2008). All these methods could in principle lead to a material with controlled delivery functionality similar to what is accomplished with the direct incorporation technique. The release dynamics can however be expected to differ. The focus of this review is primarily on controlled release based on direct incorporation according to Equation 1, although some results based on materials using other methods are also included.

\section{Dex DELIVERY—QUANTITIES AND EFFICIENCY}

The theoretically possible inclusion of Dex in a polymer film, based on the counter ion incorporation technique, can be estimated by Equation 2 (Skotheim and Reynolds, 2006):

$$
m_{\text {dopant }}=\frac{Q_{\text {dep }}}{F} \cdot \frac{\gamma M_{\text {dopant }}}{2+\gamma}
$$

$F$ stands for Faraday's constant and equals $96485 \mathrm{C} / \mathrm{mol}$ and $Q_{d e p}$ stands for deposition charge. With Dex as dopant, the commonly accepted assumption that the doping level $\gamma=0.3$, and the molecular weight of Dex, $M_{D e x}=392 \mathrm{~g} / \mathrm{mol}$, Dex inclusion per charge consumed in the electrodeposition process would be approximately $700 \mu \mathrm{g} / \mathrm{C}$. The deposition charge density that would be considered reasonable varies, depending on the stability of the polymer system, but $300 \mathrm{mC} / \mathrm{cm}^{2}$ would clearly be within the realistic range. This would mean a total included Dex mass $m_{\text {Dex }}=210 \mu \mathrm{g} / \mathrm{cm}^{2}$ according to Equation 2. Most likely higher deposition charges could be used (Li and Huang, 2007).

A handful of papers point out the Dex levels that would be required for efficient treatment of glial scarring. Based on the assumption that the volume of interest could be defined as a sphere of radius $500 \mu \mathrm{m}$ enclosing the electrode (Wadhwa et al., 2006), and that an efficient concentration would be expected to lie within the range $0.2-1 \mu \mathrm{M}$ (Golde et al., 2003; Shain et al., 2003; Zhong et al., 2005), further assuming a microelectrode radius of $\sim 15 \mu \mathrm{m}$, the electrode would need to be able to deliver a concentration of $6-30 \mu \mathrm{g} / \mathrm{cm}^{2}$ from its surface.

Considering various papers report delivery of Dex in the range $3-126 \mu \mathrm{g} / \mathrm{cm}^{2}$ in total, several single efficient doses would be possible with the presented technology (Wadhwa et al., 2006; Moulton et al., 2008; Stevenson et al., 2010; Sirivisoot et al., 
2011; Xiao et al., 2012). In light of these values one might argue that optimizing control is even more important than maximizing output per pulse or sweep.

\section{RELEASE SYSTEMS AND MECHANISMS}

The drug delivery from a conducting polymer is a result of the interplay between electrostatic interaction with the surrounding electrolyte, mechanical actuation of the film as a response to the different swelling states upon redox, and conformational changes in the polymer structure. To which extent each of the mechanisms contributes varies depending on the film morphology, the triggering signal and the use of any additional CI and must therefore be evaluated separately for each case. Three types of triggering signals are considered here namely redox sweeping, constant potential (CP) and pulsing (Figure 1). Redox sweeping is mainly referred to as the electrochemical measurement term CV.

The most simple release system would be a one-layer deposition where an anionic drug is included as a counter ion directly in the deposition process. Assuming that the drug is thereby homogenously distributed within the polymer film, and electrostatic interaction is the only responsible mechanism, the quantity of drug that is released should be proportional to the applied charge. This simplified view would imply that it is only the absolute charge transfer over the interface that matters for the quantity of drug delivered, regardless of if the charge is delivered as a pulse or a sweep. However, this is a crude simplification for a vastly more complex process (Kontturi et al., 1998; Majumdar et al., 2008). In fact, experimental data shows that the dynamics of the release signal greatly matters for the outcome.

In Table 1 a short compilation of release trigger signals and experienced results from different conducting polymer based release systems can be seen. Signals vary from steady potentials over slow CV sweeps to faster pulsed signals. From the experimental data reported in these studies it is evident that release is not solely ruled by charge transfer. Multiple ions contribute to charge transfer making the direct correlation of interfacial charge transfer to delivery of a specific species less trivial (Pyo et al., 1994; Jager et al., 2000; Pernaut and Reynolds, 2000; Li and Huang, 2007).

Some early work on PPy:ATP membranes gives important insight into the different mechanisms responsible for CP based drug delivery (Pyo et al., 1994; Pernaut and Reynolds, 2000). Based on parametric studies on inclusion and release of Adenosin Triphosphate (ATP), it was found that small, highly mobile cations are initially driven into the film and first after several minutes the release of ATP becomes the dominant process. Furthermore, even though the dissociation of the ionic drug from the polypyrrole chain may be fast, the actual release from the film is driven by diffusion and is therefore a slow process (Pernaut and Reynolds, 2000; Wadhwa et al., 2006; Li and Huang, 2007; Leprince et al., 2010). Fast signals would according to these findings mainly result in exchange of small anions/cations at the superficial layer of the polymer, for which the diffusion coefficients are low.

The majority of studies report that $\mathrm{CV}$ is vastly more efficient for driving release than CPs (Pyo et al., 1994; Pernaut and Reynolds, 2000; Wadhwa et al., 2006; Li and Huang, 2007). This underlines the complexity of the events involved in the release process far beyond what can be accounted for by the simplified electrostatic equation. The structural changes in the polymer upon redox are expected to play a major part. Most likely all the stored drug is not immediately accessible for release. Diffusion of ions within the film, and rearrangement of the polymer chains over time, exposes new deposits of drug that were not immediately accessible for the first release attempt.

Electro-actuation contributes to such conformational changes but in addition is expected to serve as a purely mechanical release mechanism for any substance entrapped within the film (Abidian et al., 2006; Thompson et al., 2006; Li and Huang, 2007). The polymer film can both shrink and swell upon reduction depending on the size of the counter ion (Jager et al., 2000). For CI in the intermediate size range such as Dex and ATP it is not straight forward to predict which mechanism would dominate. Nevertheless, repeated redox cycling will swell and shrink the film interchangeably. Actively reversing the potential at a slow rate, such as in $\mathrm{CV}$, contributes to more efficient release for the anodic cycle both through inner rearrangement and through mechanical contraction of the matrix.

In summary, the efficiency with which interfacial charge transfer can contribute to release of the intended drug depends on the dynamics of the trigger signal. Furthermore, depending on diffusivity and thickness of the individual polymer film, the

\section{Cyclic Voltammetry (CV)}

V

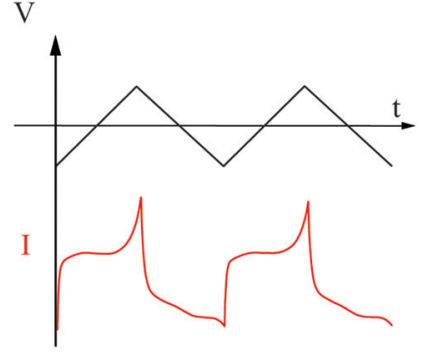

Constant potential (CP)

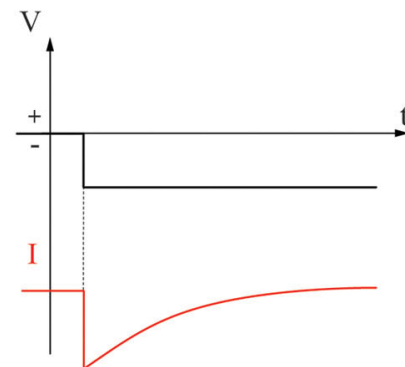

Pulsing

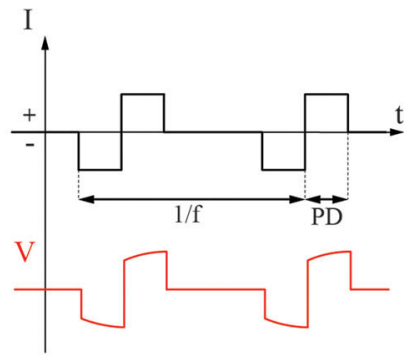

FIGURE 1 | The three different types of trigger signals for electrochemically controlled release that are discussed in the paper. The driving signal is outlined in black and the follower signal in red. 


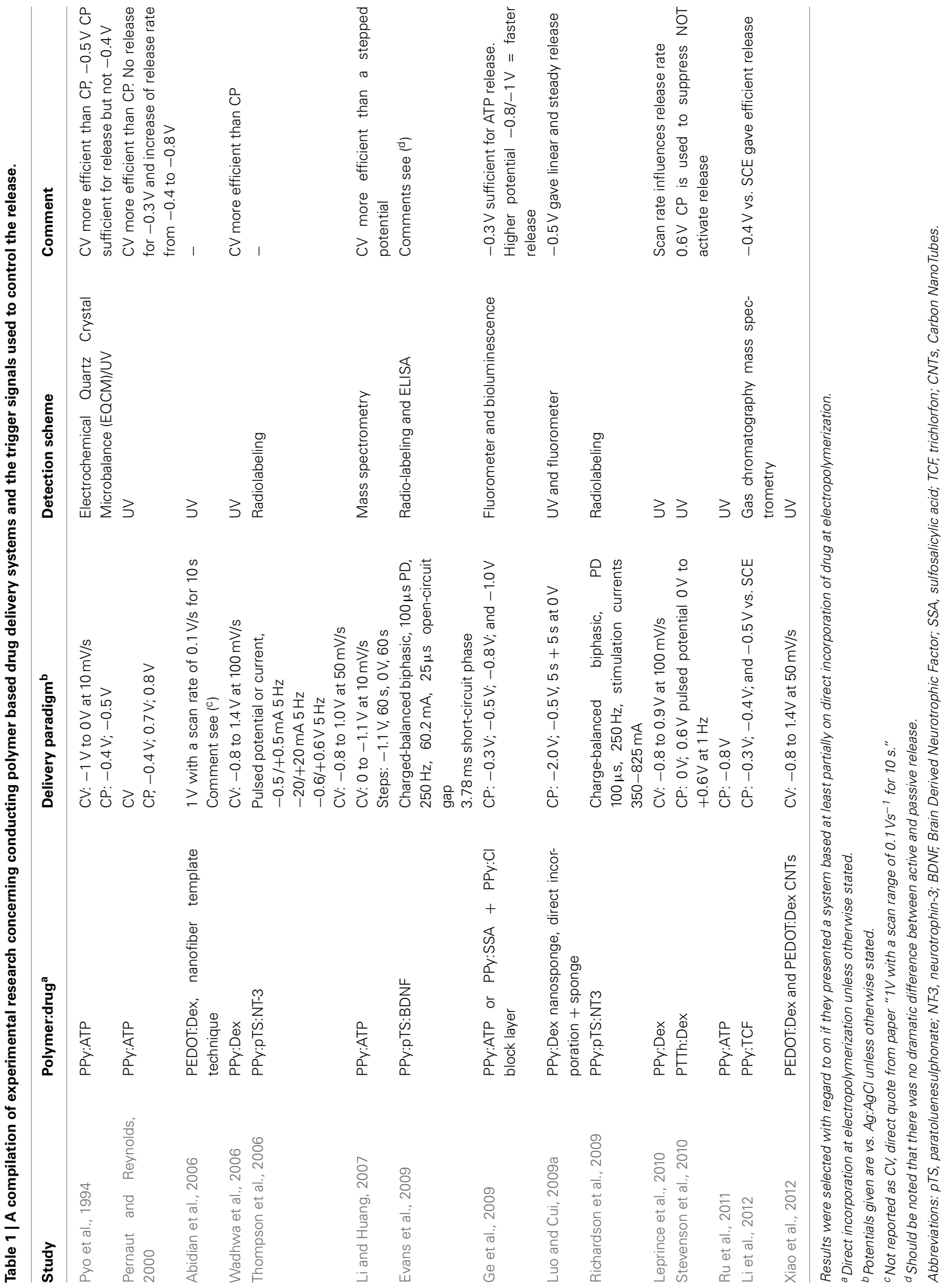


output of drug can be expected to vary. It is possible to alter the electrodeposition protocol to achieve higher diffusivity of the film, or even build layers with different properties to optimize the active vs. passive release behavior for matching a certain release protocol (Pernaut and Reynolds, 2000; Ge et al., 2009; Ru et al., 2011; Jiang et al., 2013). Moreover, the effect of the purely mechanical actuation can be further exploited by including pores in the film, for instance by a templated electrodeposition process (Abidian et al., 2006; Luo and Cui, 2009a; Luo et al., 2011). While in early work, the systems studied were single layers, advanced strategies to increase porosity of the films and boost their storage capacity are more frequently reported in recent times. In a system where entrapment is not solely based on electrostatic interaction, the mechanical actuation could be expected to be the dominant mechanism for release.

Despite the complexity of delivery mechanisms, one can make a few general assumptions on which type of signal that would be the most efficient for release from a polymer system regardless of its structure. It is clear that the theoretical arguments speak in favor for slow signals rather than fast pulsing, and especially for $\mathrm{CV}$ which would allow both mechanical actuation and electrostatics to contribute. There is a substantial risk that fast signals will not lead to efficient transfer of the intended ions. Furthermore, for a pulsed signal, the electrode will return to the open cell potential in between releases (mono-phasic pulsing) or even be actively reversed (bi-phasic pulsing). This means each delivery will be immediately followed by a signal actively driving the ionic flow in the opposite direction. This could in theory mean a reuptake of drug. However, the infiltration of small ions from solution to replace the larger ions delivered is more likely. One way to minimize the plausible reuptake of drug would be to allow the molecules additional time to diffuse away from the surface and be replaced by other ions at the reversed potential, speaking for the introduction of an interpulse delay time.

It should be noted that despite all these points speaking for active release with slow signals, release has been experimentally proven also for systems with fast pulsing (Evans et al., 2009; Richardson et al., 2009; Thompson et al., 2010). Thompson et al. do not specifically report pulse duration, only that they use biphasic stimulation delivered at a $5 \mathrm{~Hz}$ frequency. Granted that this means the full time was used for delivery of pulse trains this would however mean PDs in the range of $100 \mathrm{~ms}$ which is far from the PDs that would normally be used to trigger neural activity. The pulses reported by Evans et al. and also by Richardsson et al. come closer to real stimulation parameters with pulse widths of $100 \mu \mathrm{s}$ delivered at $250 \mathrm{~Hz}$ (Evans et al., 2009; Richardson et al., 2009). It should be mentioned that there was no dramatic difference between active and passive release in the PPy/pTS/BDNF system but clearly an effect for the PPy:pTS:NT3 system. This would speak for a release controlled by conformational changes of the polymer rather than an electrostatic driving force. The authors in addition argue for that changes in hydrophobic properties of the polymer matrix would contribute to release in this case.

The CV ranges reported vary widely. The lowest vertex potential reported is at $-1 \mathrm{~V}$ and the highest vertex potential at $1.4 \mathrm{~V}$ vs. $\mathrm{Ag}$ :AgCl. Commonly, only the anodic part of the sweep is used. It has also been pointed out that the scan rate further influences release efficiency of the single CV (Leprince et al., 2010). Sweep rates reported are in the range of $10-100 \mathrm{mV} / \mathrm{s}$. For the constant potential driven systems, the potentials required to actively drive release are in the range of -0.3 to $-0.5 \mathrm{~V}$ vs. Ag:AgCl. Clearly this will depend on the nature of the ionic drug, which conducting polymer that is used and the morphological properties of the individual polymer layer.

To some extent, a conducting polymer film that is physically degrading upon electrochemical stress can also act as a controlled delivery system. As the film falls apart, molecules that were immobilized in the structure are released at a rate that can be controlled by the level of stress. The weakest link is often the adhesion to the underlying substrate leading to complete or fragmental delamination, depending on the film cohesion. It might be difficult to judge whether a certain system performs delivery based on actuation, electrostatic interactions or simply by degradation, something that might be less favorable with an electrode intended for long term use, by only studying the release of the intended species. Ideally, one should therefore analyze if other molecules or particles are expelled from the film in parallel to the drug (Boehler and Asplund, in press). It should also be noted that conducting polymers degrade by over-oxidation meaning that the electronic structure is disrupted leading to a non-conducting material. PPy is more vulnerable to over-oxidation than PEDOT, and also the counter ion has an influence on the electronic stability (Yamato et al., 1995; Thaning et al., 2010). For this type of degradation the material itself remains at the electrode but progressive over-oxidation will influence the ion conducting properties and thereby the delivery mechanism over time.

\section{RELEASE TRIGGER SIGNALS—FROM THE SIDE OF BIOLOGICAL SAFETY}

In the previous section, the efficiency of the three release signals was discussed. However, other practical aspects such as the availability of devices capable of delivering these signals, as well as the safety for the biological environment, should naturally be taken into consideration. In the following section, the three trigger signals in Figure $\mathbf{1}$ are therefore discussed with regard to the safety of neurons.

In general, the restrictions that would apply to a signal to be applied in vivo could be summarized as follows:

1. The signal transfer should take place through reversible processes which do not lead to the formation of electrochemical by-products in the tissue or corrosive reactions at the electrode.

2. The signal must not trigger undesired activity in the neural network.

3. The signal must not induce damage to neurons.

These conditions do not completely apply for the situation where the aim is to use the signal to drive controlled release. In this case, ideally the trigger signal should be designed to be practically invisible from the side of the neuron, yet still be efficient for pushing out drugs in a reasonable time frame and with good control of the delivered amount. While restriction 1 is of the utmost importance for metallic electrodes it is not directly transferrable to polymer 
electrodes. The aim is to exchange the ionic drug in the polymer for other ions naturally available in tissue so irreversibility is to some extent here a necessity. However, the limitation of the electrode potential to prevent redox reactions in proteins and water as well as pH-shifts is a must.

It is also highly desirable that the signal used for drug release does not result in undefined excitation of surrounding neurons as in restriction 2. Normally, when evaluating how to stimulate neural tissue, the ambition is to assemble signals that are efficient in precise activation of a defined population of cells at current/pulse durations chosen to induce minimal electrochemical stress on the electrode. For the drug release approach, the signal needs to be optimized from close to the opposite perspective. The ambition would be to use a release signal that does not substantially influence surrounding cells but is efficient in pushing drugs out of the electrode.

Last, but not least, it is well-known that electrical stimulation of neurons can induce cellular injury even when the delivered stimulation is well within the boundaries given by the electrochemical safety (Shannon, 1992; Veraart et al., 2004). Normally such effects do not occur below the levels which can trigger neural activity which would ensure that a signal that confines to condition 2 is a conservative limit also for restriction 3 . However, these injury thresholds are most of the time given with consideration to the pulsing parameters that would be considered normal for neural excitation. It is therefore advisable to take a closer look at the safety boundaries from this perspective when more unconventional pulsing parameters might be put into use.

\section{PULSING}

A standard pulse for recruitment of intracortical neurons would be a biphasic train of rectangular pulses of constant current delivered at a frequency in the range of 100-400 Hz, commonly around $200 \mathrm{~Hz}$ (see Figure 1). The minimum current strength needed to excite neuronal tissue (threshold current, $I_{t h}$ ) depends on the pulse duration (PD) as in the strength-duration relationship depicted in Figure 2. PD should as a rule of thumb be chosen close to the chronaxy of the intended target tissue which in practice means durations in the range of 100-300 $\mu$ s for myelinated nerve fibers. Longer pulse durations would not be energy efficient for activation of neurons and in addition would increase the probability for non-reversible corrosive processes at the electrode site (Tehovnik, 1996).

When stimulating at PDs close to the chronaxy, typical threshold currents for neural activation would be estimated to be around $10 \mu \mathrm{A}$, but recent studies show that actual activation thresholds can be expected to be even lower than $4 \mu \mathrm{A}$ (reported for $200 \mu \mathrm{s}$ PD and $250 \mathrm{~Hz}$ ) (Tehovnik et al., 2006; Histed et al., 2009). Since the objective of the PEDOT electrode is drug delivery rather than activation, stimulation should be kept below threshold not to evoke neural activity unintentionally. In practice and according to the generally accepted current-duration relationship, this leaves two alternatives (Tehovnik, 1996). Either shortening the pulses to increase the threshold current, or use longer pulses and stay well-below the rheobase. For a pulse substantially longer than the chronaxy this means at least below $2 \mu \mathrm{A}$, preferably even lower. The longer the pulse, the more charge would be expected

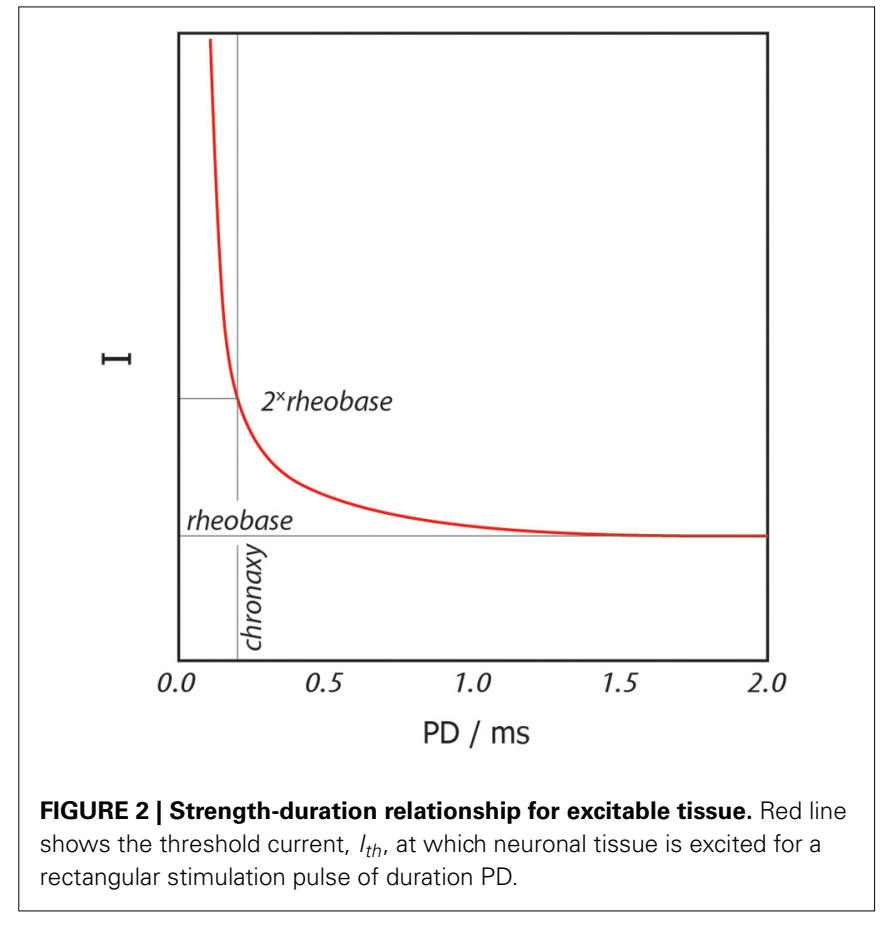

to be required to activate neurons. This clearly rather speaks for maximizing charge and thereby possibilities for ionic delivery by driving longer pulses at lower currents.

If a small and highly local amount of activated neurons can be tolerated, currents can naturally be set higher. This is something that would have to be evaluated with respect to the exact placement of the particular electrode. With knowledge of the excitability constant, $K$, of the target tissue, efficient spread of stimulation can be estimated, over the radius $r$, for a given case according to $I=K * r^{2}$ (Tehovnik, 1996; Tehovnik et al., 2006). This would provide a reasonable estimate for the volume of cells affected by a pulse if exceeding the threshold in the given case.

Tissue damage can occur as a consequence of inappropriately chosen stimulation parameters, even though the given stimulation is within the electrochemical safety limits of the electrode material. It is broadly accepted that important factors to consider are charge per phase and charge density delivered from the electrode (McCreery et al., 1990; Shannon, 1992). The mechanisms behind such effects are not completely understood but it is believed that electroporation is a main contributor to this kind of tissue damage as well as mass action phenomena, for instance depletion of oxygen or glucose, or excessive release of glutamate, caused by local neural overactivity (Merrill et al., 2005). Since signals in the case discussed here should be chosen with the aim not to induce neural signaling, only electroporation would need to be considered.

Taking also restriction 3 into account, there is reason to ask if there is a risk for the occurrence of electroporation based on this type of pulsing? A detailed insight into how the threshold for electroporation can be expected to vary with other stimulation parameters is given by Butterwick et al. (2007). One conclusion of their work is that, for longer pulses (PDs in the range of ms), the threshold current density at which electroporation can be 
expected to occur is lower than for shorter pulses. The threshold current density scales with PD approximately as 1/sqrt(PD). It is also clear from their results that with pulsing frequencies exceeding $50 \mathrm{~Hz}$ repetition frequency thresholds are significantly lower than for single shots. For electrodes smaller than $300 \mu \mathrm{m}$ it is further concluded that total current would be the main limitation, and injury thresholds would not to the same extent be influenced by current density as is seen with the larger electrodes. Their results are based on measurements in chicken retinas but correlate well to measurements in earlier work concerning injury thresholds in cortical tissue (McCreery et al., 1990; Shannon, 1992; Veraart et al., 2004). It is therefore assumed that the safe limits reported would be a reasonable figure of merit for stimulation of any target tissue in the central as well as in the peripheral nervous system.

The lowest threshold where stimulation induced damage was detected in their experiments was determined to be $61 \mathrm{~mA} / \mathrm{cm}^{2}$ at a pulse duration of $6 \mathrm{~ms}$ for repetitive pulsing $(>50 \mathrm{~Hz})$. Translated to a microelectrode of $50 \mu \mathrm{m}$ that would correspond to a charge per pulse of $7.2 \mathrm{nC}$. However, for pulses shorter than the ms, threshold currents are substantially higher. Deduced from the data presented by Butterwick, for the $600 \mu \mathrm{s}$, expected thresholds would rather be in the range of $0.2 \mathrm{~A} / \mathrm{cm}^{2}$. Due to the shorter pulse length this would in fact mean a lower charge per phase threshold, for the $50 \mu \mathrm{m}$ electrode $2.3 \mathrm{nC} / \mathrm{ph}$ ase. This would speak for that electroporation could occur even with a minor elevation of the stimulation current.

The study also presents results regarding the influence of the size of the electrode and the values cited above relate to a large electrode, not a microelectrode. If taking their results concerning microelectrodes into account, here smaller than $300 \mu \mathrm{m}$ in diameter, the threshold charge per phase that could safely be delivered did in fact not depend on electrode size. The smallest electrode used in their experiment was $\sim 100 \mu \mathrm{m}$ in diameter and the threshold, when investigated on the retina cells using $600 \mu$ s single pulses, were found to be $\sim 70 \mathrm{nC}$ per phase. This threshold should, according to their theory of size independence, also be valid for smaller electrodes. Even though these particular results describe thresholds for one single pulse, there is substantial margin to the $1.2 \mathrm{nC}$ given by the $2 \mu \mathrm{A}$ and $600 \mu$ s suggested as suitable sub-threshold stimulation (second paragraph, section Pulsing).

In summary, also from the perspective of restriction 3, it would be recommendable to work with PDs longer than the chronaxy. It further appears as if PDs are on the longer side, and threshold current is estimated to be below the rheobase, there is no substantial risk for disruption of cellular membrane integrity as a consequence of the pulsed trigger signal.

In order to establish boundaries for safety it is of interest to glance at the field of deep brain stimulation (DBS), where intracortical stimulation has been therapeutically used over more than a decade. The technology is FDA approved and one could thereby argue that these stimulation protocols have already been proven safe for use in patients. Kern and Kumar (2007) report common values for the Sub Thalamic Nucleus (STN) DBS to be in the range $130-185 \mathrm{~Hz}$ with typical pulse durations of $60 \mu \mathrm{s}$. However, the extremely short pulse durations used for DBS are most likely not efficient for drug expulsion. Release relies to large extent on slower processes as has already been discussed in the previous section.

\section{CYCLIC VOLTAMMETRY}

It has already been pointed out that slow redox sweeping of the electrode has been found efficient for delivery of ionic drugs. The stimulation effects of CV in brain tissue are not specifically addressed in the literature. Theoretically, if the current-duration curves are extrapolated into infinitely long pulses, which would be a reasonable approximation for the smooth transitions of a $\mathrm{CV}$ curve, the suggestion would be that as long as the current is still below the rheobase the neurons would not be excited.

If the neuronal activity is not directly influenced, the restrictions would rather be set by the possibly detrimental electrochemical reactions that could occur as a by-product of any excessive voltage. The two parameters to consider are the vertex potentials and the sweep rate. The non-conservative constraints for the vertex potentials would be given by the water window $(-0.6 \mathrm{~V}$ to $0.8 \mathrm{~V}$ vs. $\mathrm{Ag}: \mathrm{AgCl})$ since the evolution of hydrogen $(-0.6 \mathrm{~V})$ and oxygen $(0.8 \mathrm{~V})$, respectively, would occur at the electrode upon exceeding this window. Over-oxidation of PEDOT would not be expected to occur at voltages lower than $1.1 \mathrm{~V}$ and is therefore not a limiting factor in this case. This is however not sufficient to support that no other electrochemical reactions of importance take place within this window. Furthermore, appropriate reference electrodes are often not available in the implanted situation meaning that possible variations in applied voltages must be taken into account. Materials do either not deliver a stable potential in chronic implantations or biofouling clogs pores in ion-selective membranes.

One example on actual parameters used in a similar biological setting can be found in recent work monitoring electrochemical characteristics of implanted electrodes. Kane et al. (2011) employs the full water window at a sweep rate of $50 \mathrm{mV} / \mathrm{s}$ for their in vivo $\mathrm{CV}$ investigations and do not report any adverse tissue response as a consequence. In the sensor literature, fast CV is often suggested for instance for in vivo monitoring of neurotransmitters. It should be noted that so far sweep rates used are in the range of $300 \mathrm{~V} / \mathrm{s}$ and thus far beyond what has been proposed for drug release (Pihel et al., 1996). The biological response was not the primary target for the analysis in either study and was therefore not carefully observed. Scans used for analytical purposes in an experiment with a sedated animal, and limited to a few occasions, are not necessarily suitable for repeated use in proximity of a population of highly sensitive cells. In both studies animals were anaesthetized which might not be possible to perform on a bi-weekly to weekly basis needed to support efficient drug release.

\section{BIAS POTENTIAL}

A bias potential of approximately $-0.5 \mathrm{~V}$ vs. $\mathrm{Ag}$ : $\mathrm{AgCl}$ could be used to drive drug release. Cells are not expected to respond adversely to a steady potential if kept within reasonable boundaries. For instance, a positive interpulse bias potential of $0.6 \mathrm{~V}$ has been suggested as a method for boosting performance of iridium oxide electrodes and has been applied to SIROF coatings in vivo (Cogan et al., 2006; Kane et al., 2011). The authors comment that the long-term consequences of the low net current needed to 
maintain the bias potential has not yet been explored. However, for the drug release, the bias potential would only be applied at the specific occasions where drug release is requested which qualifies as a limited time frame and should be safe for use.

\section{DISCUSSION}

\section{OTHER RELEASE STRATEGIES AND DRUGS}

Apart from Dex, also various other anti-inflammatory drugs like $\alpha-\mathrm{MSH}, \mathrm{IL}-1-\mathrm{RA}$, or heparin have been proposed in literature for treatment of inflammatory reactions. These molecules target different receptor pathways in the inflammation cascade and can thus be interesting alternatives or complements to the Dex (Bridges and Garcia, 2008; Go et al., 2012). All of the listed molecules are typically delivered from passively eluting systems due to their chemical properties and overall size, which makes them incompatible with the ion-exchange principle provided by conducting polymers. In contrast, Dex is in the appropriate size range $(392 \mathrm{Da})$ and features an anionic charge characteristic, which makes is eligible for both passive and actively controlled release systems. Furthermore, Dex is known to be the most potent glucocorticoid drug for anti-inflammatory treatment due to its effect in multiple receptor pathways, which reveals this drug as first choice for the realization of an anti-inflammatory drug eluting system for treatment of the glial scar. For a more detailed description of relevant molecules and their impact on the adverse host response to implanted biomedical devices the excellent review by Bridges et al. is strongly recommended (Bridges and Garcia, 2008). It should be mentioned that it would be possible to use a deposition technique not relying on direct incorporation to combine the active delivery approach also with these substances (Abidian et al., 2006; Luo et al., 2011).

It is a valid question to ask how the actively controlled release of Dex from a microelectrode would compare to a delivery coating passively leaking Dex into tissue, or systemic treatment (Shain et al., 2003; Spataro et al., 2005; Zhong and Bellamkonda, 2007). The latter has the clear downside of requiring much higher total doses distributed to reach an efficient concentration at the point of interest and side-effects would thus be a main concern. In situ delivery, avoiding the counterproductive neuroendocrine feedbacks, is from this perspective clearly beneficial regardless of if the system is based on a passive or active release approach.

The active release system offers controllability but for a limited quantity whereas the passive system can cover the complete surface of a probe, thereby storing a much larger quantity, but hold few options to influence release dynamics. From the data available in literature it is difficult to judge whether such a system would be more successful in mitigating inflammation than the actively triggered release approach. The answer to this question could only be given if the necessary time course of the treatment was known. It is still an open question whether the anti-inflammatory treatment over the time course of a month, which would be the critical period in which the scar forms, influences the state of the surrounding tissue also in the long term (Szarowski et al., 2003; Biran et al., 2005; Potter et al., 2012). If it does, the lower quantity delivered by the microelectrodes might still be sufficient, meaning higher drug load is not a substantial benefit but could be traded for more precise release control. On the other hand, treatment might be needed for a much longer time period which would make the opportunity to load a higher quantity of drug much more attractive. A certain control of release dynamics in the passive release system could still be accomplished by carefully tailoring the degradability properties of the release matrix. In theory, nothing speaks against that such a system in the future could include certain responsiveness to biological factors, for instance react to chemical factors present for a higher degree of inflammation, by increasing the release rate.

It should be noted that such a delivery matrix, which can act as the golden standard for passive release, does not yet exist. Thus, the passive release system relies on substantial development in this direction in the same way as the polymer electrodes hold room for future optimization. Recent result show that also surface immobilized Dex might have an effect for improving probe integration which illustrates the need for further work to elucidate the underlying mechanisms involved in the tissue response to Dex (Grand et al., 2010). In summary, the passive delivery approach should be pursued in parallel with the active delivery systems, as an interesting alternative or even additional approach, since the use of the one delivery system in fact does not exclude the use of the other as a complement.

\section{RELEASE TRIGGER SIGNALS-CONCLUDING REMARKS}

Taking all the considerations regarding efficiency and safety into account they all speak in favor for slow release signals. CV seems to be the most suitable from the efficiency perspective and most likely a moderate use of voltammetric sweeping will not be harmful to tissue or lead to undeliberate excitation of the neuronal network. This however needs to be confirmed in experiments.

In practice, the equipment needed to perform well-controlled $\mathrm{CV}$ at the implant site might however not always be available. Proper CV measurements require a three-electrode electrochemical control system and the technologies for on-probe integration of stable reference electrodes on implants are not yet established (Tolosa et al., 2013).

Considering this practical aspect, it might therefore still be the most convenient to use a pulsed signal for release rather than a sweep. In this case it would be advisable to aim for low currents and PDs significantly longer than the chronaxy. Furthermore, to present as little stress as possible to the tissue, low pulsing frequencies should be used. Lowering the pulsing frequency and compensating by extending the overall stimulation time should have little effect on the release itself but would allow higher currents before stimulation induced damage to neurons can be expected to occur (Butterwick et al., 2007). Charge balance is not a necessity for this kind of drug release. On one hand it might contribute to increased actuation, on the other hand it might be counterproductive if the Dex ions are immediately retrieved by the reversed pulse. An interpulse delay could mitigate the second effect.

One possibility to, with a simple device, imitate the effect of a $\mathrm{CV}$ is to exchange the sweep with interchangeable voltage steps, set to the upper and lower vertex potential. Microsized devices capable of maintaining a fixed voltage vs. a reference, and suitable for implantation, have been described by others (Islam et al., 2010). In Figure 3, the current response of a PEDOT:Dex 
electrode to a cyclic voltammogram can be seen as well as the current response to a rectangular pulse. It is clear that the current flow follows another scheme than in the carefully controlled slow $\mathrm{CV}$. What this in practice would mean for the drug release should be further investigated experimentally.

Finally, not only efficiency of release but also controllability should be considered. For the application, it is desirable that a precise dose of Dex can be delivered at each attempt, which means the response to the trigger signal must be predictable. Taking the complexity of events involved in release into account, such one to one correlation is not trivial to accomplish. More likely, the response of the polymer will change over time and with each delivery attempt, something that must be carefully considered to ensure the trigger signal has the intended effect throughout the full experimental time frame. This needs to be further investigated before a specific polymer delivery system is put into use.

Since the drug delivery systems reviewed here are intended for local treatment targeting only the cells in the immediate vicinity of the electrode, it is highly desirable that the very same electrode used for delivery can still function as a neural interface. For recording this is not expected to be a problem since both PEDOT and PPy are known for their low impedance in this respect. Although the drug delivery electrodes in general are outperformed by the regular surfactant based conducting polymer materials in terms of impedance, it has repeatedly been shown that also drug containing conducting polymer electrodes still lower the impedance in comparison to a bare metallic surface (Wadhwa et al., 2006; Luo et al., 2011).
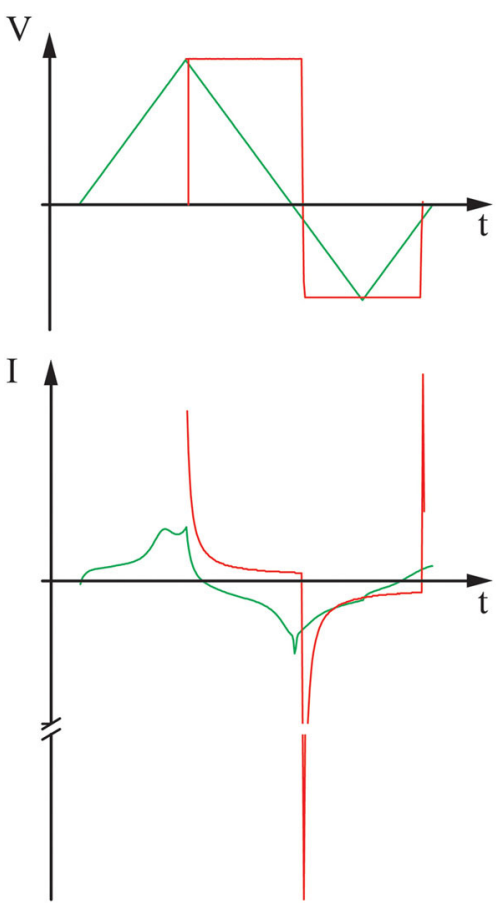

FIGURE 3 | A comparison between the current response to a swept voltage vs. a stepped voltage for a PEDOT:Dex delivery system. Measurement was performed in PBS and vs. an $\mathrm{Ag}: \mathrm{AgCl}$ reference.
For stimulation the central question would be if stimulation can be performed without unintentionally triggering release? From the literature reviewed here it is not possible to make a conclusive statement on whether this would at all be possible. As discussed, some authors do indeed present release based on the type of fast pulsing that would be common to use for stimulation purposes (Evans et al., 2009; Richardson et al., 2009). However, it can be concluded that slow sweeps are vastly more effective for drug release (Table 1) and several authors report that they need to reach a certain voltage before actual release is triggered (e.g., Pyo et al., 1994; Pernaut and Reynolds, 2000). It is plausible that smart materials design can further exploit this effect making the release-free stimulation possible within certain boundaries.

\section{REFERENCES}

Abidian, M. R., Kim, D. H., and Martin, D. C. (2006). Conducting-polymer nanotubes for controlled drug release. Adv. Mater. 18, 405-409. doi: 10.1002/adma.200501726

Asplund, M., Von Holst, H., and Inganas, O. (2008). Composite biomolecule/PEDOT materials for neural electrodes. Biointerphases 3, 83-93. doi: 10.1116/1.2998407

Biran, R., Martin, D. C., and Tresco, P. A. (2005). Neuronal cell loss accompanies the brain tissue response to chronically implanted silicon microelectrode arrays. Exp. Neurol. 195, 115-126. doi: 10.1016/j.expneurol.2005. 04.020

Bobacka, J., Lewenstam, A., and Ivaska, A. (2000). Electrochemical impedance spectroscopy of oxidized poly(3,4-ethylenedioxythiophene) film electrodes in aqueous solutions. J. Electroanal. Chem. 489, 17-27. doi: 10.1016/S00220728(00)00206-0

Boehler, C., and Asplund, M. (in press). A detailed insight into drug delivery from PEDOT based on analytical methods: effects and side-effects. J. Biomed. Mater. Res. A.

Bridges, A. W., and Garcia, A. J. (2008). Anti-inflammatory polymeric coatings for implantable biomaterials and devices. J. Diabetes Sci. Technol. 2, 984-994.

Butterwick, A., Vankov, A., Huie, P., Freyvert, Y., and Palanker, D. (2007). Tissue damage by pulsed electrical stimulation. IEEE Trans. Biomed. Eng. 54, 2261-2267. doi: 10.1109/TBME.2007.908310

Cogan, S. F., Troyk, P. R., Ehrlich, J., Plante, T. D., and Detlefsen, D. E. (2006). Potential-biased, asymmetric waveforms for charge-injection with activated iridium oxide (AIROF) neural stimulation electrodes. IEEE Trans. Biomed. Eng. 53, 327-332. doi: 10.1109/tbme.2005.862572

Evans, A. J., Thompson, B. C., Wallace, G. G., Millard, R., O’leary, S. J., Clark, G. M., et al. (2009). Promoting neurite outgrowth from spiral ganglion neuron explants using polypyrrole/BDNF-coated electrodes. J. Biomed. Mater. Res. A 91A, 241-250. doi: 10.1002/jbm.a.32228

Ge, D., Tian, X., Qi, R., Huang, S., Mu, J., Hong, S., et al. (2009). A polypyrrolebased microchip for controlled drug release. Electrochim. Acta 55, 271-275. doi: 10.1016/j.electacta.2009.08.049

Go, D. P., Palmer, J. A., Gras, S. L., and O'connor, A. J. (2012). Coating and release of an anti-inflammatory hormone from PLGA microspheres for tissue engineering. J. Biomed. Mater. Res. A 100A, 507-517. doi: 10.1002/jbm.a. 33299

Golde, S., Coles, A., Lindquist, J. A., and Compston, A. (2003). Decreased iNOS synthesis mediates dexamethasone-induced protection of neurons from inflammatory injury in vitro. Eur. J. Neurosci. 18, 2527-2537. doi: 10.1046/j.14609568.2003.02917.x

Grand, L., Wittner, L., Herwik, S., Gothelid, E., Ruther, P., Oscarsson, S., et al. (2010). Short and long term biocompatibility of NeuroProbes silicon probes. J. Neurosci. Methods 189, 216-229. doi: 10.1016/j.jneumeth.2010. 04.009

Histed, M. H., Bonin, V., and Reid, R. C. (2009). Direct activation of sparse, distributed populations of cortical neurons by electrical microstimulation. Neuron 63, 508-522. doi: 10.1016/j.neuron.2009.07.016

Islam, A. B., Haider, M. R., Atla, A., Islam, S. K., Croce, R., Vaddiraju, S., et al. (2010). "A potentiostat circuit for multiple implantable electrochemical 
sensors," in Electrical and Computer Engineering (ICECE), 2010 International Conference, (Wuhan), 314-317.

Jager, E. W. H., Smela, E., and Inganas, O. (2000). Microfabricating conjugated polymer actuators. Science 290, 1540-1545. doi: 10.1126/science.290. 5496.1540

Jiang, S., Sun, Y., Cui, X., Huang, X., He, Y., Ji, S., et al. (2013). Enhanced drug loading capacity of polypyrrole nanowire network for controlled drug release. Synth. Met. 163, 19-23. doi: 10.1016/j.synthmet.2012.12.010

Kane, S. R., Cogan, S. F., Ehrlich, J., Plante, T. D., and McCreery, D. B. (2011). Electrical performance of penetrating microelectrodes chronically implanted in cat cortex. Conf. Proc. IEEE Eng. Med. Biol. Soc. 2011, 5416-5419. doi: 10.1109/iembs.2011.6091339

Kern, D. S., and Kumar, R. (2007). Deep brain stimulation. Neurologist 13, 237-252. doi: 10.1097/NRL.0b013e3181492c48

Kontturi, K., Pentti, P., and Sundholm, G. (1998). Polypyrrole as a model membrane for drug delivery. J. Electroanal. Chem. 453, 231-238. doi: 10.1016/s00220728(98)00246-0

Leprince, L., Dogimont, A., Magnin, D., and Demoustier-Champagne, S. (2010). Dexamethasone electrically controlled release from polypyrrole-coated nanostructured electrodes. J. Mater. Sci. Mater. Med. 21, 925-930. doi: 10.1007/s10856-010-4008-6

Li, L. D., and Huang, C. B. (2007). Electrochemical/electrospray mass spectrometric studies of electrochemically stimulated ATP release from PP/ATP films. J. Am. Soc. Mass Spectrom. 18, 919-926. doi: 10.1016/j.jasms.2007.01.015

Li, Y., Ewen, R. J., Campbell, S. A., and Smith, J. R. (2012). Electrochemically controlled release of antischistosomiasis agents from polypyrrole. J. Mater. Chem. 22, 2687-2694. doi: 10.1039/c2jm15298c

Luo, X., and Cui, X. T. (2009a). Sponge-like nanostructured conducting polymers for electrically controlled drug release. Electrochem. commun. 11, 1956-1959. doi: 10.1016/j.elecom.2009.08.027

Luo, X., Matranga, C., Tan, S., Alba, N., and Cui, X. T. (2011). Carbon nanotube nanoreservior for controlled release of anti-inflammatory dexamethasone. Biomaterials 32, 6316-6323. doi: 10.1016/j.biomaterials.2011.05.020

Luo, X. L., and Cui, X. T. (2009b). Electrochemically controlled release based on nanoporous conducting polymers. Electrochem. Commun. 11, 402-404. doi: 10.1016/j.elecom.2008.11.052

Majumdar, S., Kargupta, K., and Ganguly, S. (2008). Mathematical modeling for the ionic inclusion process inside conducting polymer-based thin-films. Polym. Eng. Sci. 48, 2229-2237. doi: 10.1002/pen.21170

McCreery, D. B., Agnew, W. F., Yuen, T. G., and Bullara, L. (1990). Charge density and charge per phase as cofactors in neural injury induced by electrical stimulation. IEEE Trans. Biomed. Eng. 37, 996-1001. doi: 10.1109/10.102812

Merrill, D. R., Bikson, M., and Jefferys, J. G. (2005). Electrical stimulation of excitable tissue: design of efficacious and safe protocols. J. Neurosci. Methods 141, 171-198. doi: 10.1016/j.jneumeth.2004.10.020

Moulton, S. E., Imisides, M. D., Shepherd, R. L., and Wallace, G. G. (2008). Galvanic coupling conducting polymers to biodegradable $\mathrm{Mg}$ initiates autonomously powered drug release. J. Mater. Chem. 18, 3608-3613. doi: 10.1039/B805481a

Pernaut, J. M., and Reynolds, J. R. (2000). Use of conducting electroactive polymers for drug delivery and sensing of bioactive molecules. A redox chemistry approach. J. Phys. Chem. B 104, 4080-4090. doi: 10.1021/ jp994274o

Pihel, K., Walker, Q. D., and Wightman, R. M. (1996). Overoxidized polypyrrolecoated carbon fiber microelectrodes for dopamine measurements with fast-scan cyclic voltammetry. Anal. Chem. 68, 2084-2089. doi: 10.1021/ ac960153y

Potter, K. A., Buck, A. C., Self, W. K., and Capadona, J. R. (2012). Stab injury and device implantation within the brain results in inversely multiphasic neuroinflammatory and neurodegenerative responses. J. Neural Eng. 9:046020. doi: $10.1088 / 1741-2560 / 9 / 4 / 046020$

Pyo, M., Maeder, G., Kennedy, R. T., and Reynolds, J. R. (1994). Controlled release of biological molecules from conducting polymer modified electrodes: The potential dependent release of adenosine 5'-triphosphate from poly(pyrrole adenosine 5'-triphosphate) films. J. Electroanal. Chem. 368, 329-332. doi: 10.1016/0022-0728(93)03071-V

Richardson, R. T., Wise, A. K., Thompson, B. C., Flynn, B. O., Atkinson, P. J., Fretwell, N. J., et al. (2009). Polypyrrole-coated electrodes for the delivery of charge and neurotrophins to cochlear neurons. Biomaterials 30, 2614-2624. doi: 10.1016/j.biomaterials.2009.01.015
Ru, X. N., Shi, W., Huang, X., Cui, X., Ren, B., and Ge, D. T. (2011). Synthesis of polypyrrole nanowire network with high adenosine triphosphate release efficiency. Electrochim. Acta 56, 9887-9892. doi: 10.1016/j.electacta.2011.08.063

Shain, W., Spataro, L., Dilgen, J., Haverstick, K., Retterer, S., Isaacson, M., et al. (2003). Controlling cellular reactive responses around neural prosthetic devices using peripheral and local intervention strategies. IEEE Trans. Neural Syst. Rehabil. Eng. 11, 186-188. doi: 10.1109/TNSRE.2003.814800

Shannon, R. V. (1992). A model of safe levels for electrical stimulation. IEEE Trans. Biomed. Eng. 39, 424-426. doi: 10.1109/10.126616

Sirivisoot, S., Pareta, R., and Webster, T. J. (2011). Electrically controlled drug release from nanostructured polypyrrole coated on titanium. Nanotechnology 22:085101. doi: 10.1088/0957-4484/22/8/085101

Skotheim, T. A., and Reynolds, J. R. (2006). Conjugated Polymers: Theory, Synthesis, Properties and Characterization. Boca Raton: CRC Press.

Spataro, L., Dilgen, J., Retterer, S., Spence, A. J., Isaacson, M., Turner, J. N., et al. (2005). Dexamethasone treatment reduces astroglia responses to inserted neuroprosthetic devices in rat neocortex. Exp. Neurol. 194, 289-300. doi: 10.1016/j.expneurol.2004.08.037

Stevenson, G., Moulton, S. E., Innis, P. C., and Wallace, G. G. (2010). Polyterthiophene as an electrostimulated controlled drug release material of therapeutic levels of dexamethasone. Synth. Met. 160, 1107-1114. doi: 10.1016/j.synthmet.2010.02.035

Szarowski, D. H., Andersen, M. D., Retterer, S., Spence, A. J., Isaacson, M., Craighead, H. G., et al. (2003). Brain responses to micro-machined silicon devices. Brain Res. 983, 23-35. doi: 10.1016/S0006-8993(03)03023-3

Tehovnik, E. J. (1996). Electrical stimulation of neural tissue to evoke behavioral responses. J. Neurosci. Methods 65, 1-17. doi: 10.1016/0165-0270(95)00131-X

Tehovnik, E. J., Tolias, A. S., Sultan, F., Slocum, W. M., and Logothetis, N. K. (2006). Direct and indirect activation of cortical neurons by electrical microstimulation. J. Neurophysiol. 96, 512-521. doi: 10.1152/jn.00126.2006

Thaning, E. M., Asplund, M. L., Nyberg, T. A., Inganas, O. W., and von Holst, H. (2010). Stability of poly(3,4-ethylene dioxythiophene) materials intended for implants. J. Biomed. Mater. Res. B Appl. Biomater. 93, 407-415. doi: 10.1002/ jbm.b.31597

Thompson, B. C., Chen, J., Moulton, S. E., and Wallace, G. G. (2010). Nanostructured aligned CNT platforms enhance the controlled release of a neurotrophic protein from polypyrrole. Nanoscale 2, 499-501. doi: $10.1039 / \mathrm{b} 9 \mathrm{nr} 00259 \mathrm{f}$

Thompson, B. C., Moulton, S. E., Ding, J., Richardson, R., Cameron, A., O'leary, S., et al. (2006). Optimising the incorporation and release of a neurotrophic factor using conducting polypyrrole. J. Control. Release 116, 285-294. doi: 10.1016/j.jconrel.2006.09.004

Tolosa, V. M., Wassum, K. M., Maidment, N. T., and Monbouquette, H. G. (2013). Electrochemically deposited iridium oxide reference electrode integrated with an electroenzymatic glutamate sensor on a multi-electrode arraymicroprobe. Biosensors Bioelectron. 42, 256-260. doi: dx.doi.org/10.1016/j.bios.2012. 10.061

Turner, J. N., Shain, W., Szarowski, D. H., Andersen, M., Martins, S., Isaacson, M., et al. (1999). Cerebral astrocyte response to micromachined silicon implants. Exp. Neurol. 156, 33-49. doi: 10.1006/exnr.1998.6983

Veraart, C., Duret, F., Brelén, M., Oozeer, M., and Delbeke, J. (2004). Vision rehabilitation in the case of blindness. Expert Rev. Med. Devices 1, 139-153. doi: 10.1586/17434440.1.1.139

Wadhwa, R., Lagenaur, C. F., and Cui, X. T. (2006). Electrochemically controlled release of dexamethasone from conducting polymer polypyrrole coated electrode. J. Control. Release 110, 531-541. doi: 10.1016/j.jconrel.2005.10.027

Xiao, Y., Che, J., Li, C. M., Sun, C. Q., Chua, Y. T., Lee, V. S., et al. (2007). Preparation of nano-tentacle polypyrrole with pseudo-molecular template for ATP incorporation. J. Biomed. Mater. Res. A 80, 925-931. doi: 10.1002/jbm.a.31001

Xiao, Y., Ye, X., He, L., and Che, J. (2012). New carbon nanotube-conducting polymer composite electrodes for drug delivery applications. Polym. Int. 61, 190-196. doi: 10.1002/pi.3168

Yamato, H., Ohwa, M., and Wernet, W. (1995). Stability of polypyrrole and poly(3,4-ethylene dioxythiophene) for biosensor application. J. Electroanal. Chem. 397, 163-170. doi: 10.1016/0022-0728(95)04156-8

Yue, Z., Moulton, S. E., Cook, M., O'leary, S., and Wallace, G. G. (2013). Controlled delivery for neuro-bionic devices. Adv. Drug Deliv. Rev. 65, 559-569. doi: 10.1016/j.addr.2012.06.002 
Zhong, Y. H., and Bellamkonda, R. V. (2007). Dexamethasone-coated neural probes elicit attenuated inflammatory response and neuronal loss compared to uncoated neural probes. Brain Res. 1148, 15-27. doi: 10.1016/j.brainres.2007.02.024

Zhong, Y., McConnell, G. C., Ross, J. D., Deweerth, S. P., and Bellamkonda, R. V. (2005). "A novel dexamethasone-releasing, anti-inflammatory coating for neural implants," in Neural Engineering, 2005. Conference Proceedings. 2nd International IEEE EMBS Conference (Arlington), 522-525.

Conflict of Interest Statement: The authors declare that the research was conducted in the absence of any commercial or financial relationships that could be construed as a potential conflict of interest.
Received: 30 January 2014; accepted: 02 April 2014; published online: 13 May 2014. Citation: Asplund M, Boehler C and Stieglitz T (2014) Anti-inflammatory polymer electrodes for glial scar treatment: bringing the conceptual idea to future results. Front. Neuroeng. 7:9. doi: 10.3389/fneng.2014.00009

This article was submitted to the journal Frontiers in Neuroengineering.

Copyright (c) 2014 Asplund, Boehler and Stieglitz. This is an open-access article distributed under the terms of the Creative Commons Attribution License (CC BY). The use, distribution or reproduction in other forums is permitted, provided the original author(s) or licensor are credited and that the original publication in this journal is cited, in accordance with accepted academic practice. No use, distribution or reproduction is permitted which does not comply with these terms. 